\title{
A Novel Smart ECO Model for Energy Consumption Optimization
}

\author{
Reza Malekian ${ }^{1}$, Dijana Capeska Bogatinoska ${ }^{2}$, Aleksandar Karadimce ${ }^{2}$, Ning $\mathrm{Ye}^{3}$, Jasna Trengoska ${ }^{2}$, \\ William Asiama Nyako ${ }^{2}$ \\ ${ }^{I}$ Department of Electrical, Electronic and Computer Engineering, University of Pretoria, \\ Pretoria, South Africa, 0002 \\ ${ }^{2}$ Department of Computer Science and Engineering, University for Information Science and \\ Technology "St. Paul the Apostle", \\ Ohrid, Republic of Macedonia \\ ${ }^{3}$ Department of Computer Science and Technology, Nanjing University of Posts and \\ Telecommunications, Nanjing, P.R. China \\ reza.malekian@ieee.org
}

\begin{abstract}
Today we see various small automated devices that are used to regulate the energy consumption in households. Most of these devices work autonomously on a specific set of appliances or only for specific necessities, such as heating or cooling. The proposed smart energy solutions are focused on gathering datasets of the historical data for energy consumption and then propose different algorithms for analysing these datasets.

In this paper, we investigate the possibility of using a new Smart-ECO model, consisting of a smart device, which is running its own smart algorithm. We will conduct analysis on real-time household dataset, in order to optimize the electricity consumption per appliance. The proposed model is based on the custom preferences and behavioural habits of the people that live in the household and the inter-dependency of the appliances that are active at the moment.
\end{abstract}

Index Terms-Smart homes, regression analysis, artificial bee's colony algorithm, habitual average.

\section{INTRODUCTION}

The global population continues to grow at a steady pace, and at the same time there is a growing trend of migrating to cities. Cities represent three quarters of energy consumptions and $80 \%$ of $\mathrm{CO} 2$ emission worldwide [1]. This increasing global population implies the need for cities to get smarter in handling this large-scale urbanization. New ways should be invented to manage cities, make them more effective, improve quality of life and reduce the negative impact on the environment. Cities become smart, providing good infrastructure, and various citizen centric services; they are intersections between competitiveness, capital and sustainability. The world is ready to welcome a new wave of intelligence into our homes and workplaces.

Sustainable energy management is tied in with

Manuscript received December 29, 2014; accepted June 2, 2015

This research was funded by a grant (No. AOX220) from the National Research Foundation, South Africa in conjunction with Research Development Programme of the University of Pretoria. This research was performed in cooperation with the Institution. infrastructure and sustainability standards. Energy management will contribute highly to the quality of life for the urban population. Thus, championing the idea of energy management and investing in "smart city" technologies is the key to economic competitiveness for any city.

Understandably, smart homes contribute significantly towards smarter cities. Energy management in smart homes is carried out through smart devices, used in homes to interconnect all home appliances in order to collect data about their energy usage. A key challenge is to extract meaningful values from all the collected data. For investigation, statistics and also analysis on energy consumption data, data mining techniques can be applied This can be done while having in mind that some additional factors such as the weather or the user's behaviour patterns, can also have significant influence on the overall energy consumption.

In this paper we propose a modified Bee's algorithm, based on user's behaviour patterns, which is essential in defining habits in order to derive rules about the usage of home appliances. The Smart Electrical Consumption Optimization algorithm (Smart-ECO algorithm), as we name it, has the capability to learn from the historical patterns about the usage habits of the residents in the household. In this paper we also introduce the new concept of "habitual average" or average per habit, as a central concept. The Smart ECO algorithm will be deployed on smart devices and used for unobtrusive energy monitoring and management in the households.

The paper is organized as follows. In Section II we discuss the energy management process. In Section III, we give an overview of advanced techniques for analysis of data. In Section IV, we provide the description of the new Smart ECO model. Conclusions are given in Section V.

\section{Energy MANAGEMENT PRocess}

From the aspect of energy saving, "energy management is the process of monitoring, controlling, and conserving 
energy in a building" [2]. It is the means to control and reduce energy consumption in buildings, mainly to reduce the cost. That will highly contribute to the global aspiration to save energy and to reduce carbon emissions and consequently, the environmental damage caused by them.

This process involves a few steps:

Step 1: Measuring the energy usage and collecting the data;

Step 2: Make a data analysis in order to find and quantify possibilities to save energy;

Step 3: Analysing the collected data to see are there any improvements in energy saving;

Step 4: Repeat (go to Step 1) until a stopping criterion is met.

For metering the energy consumption data, digital devices like interval-metering systems are used. This technology delivers the data, consisting of a timestamp plus a measured value, from a metering system to an IT-system. The data is then cleaned and manipulated for analytical purposes; this is done taking into account the huge volumes of data. Different techniques for data analyses, like basic statistical analyses or data mining techniques can be applied on the dataset, to extract valid information about data. As well as that, "energy guzzlers", such as certain household appliances, can be quickly identified.

Different factors, like weather conditions or people's behaviour, have influence on the energy consumption. Weather conditions are important for the appliances that are used for heating or cooling of households. The energy usage in this case is closely correlated with the weather conditions, in this case outside air temperature.

The awareness of energy consumption can reduce electricity usage. Just small changes in the behaviour of all members of the household, can lead to significant conservation of the energy. Combination of information and goal setting strategies is able to motivate individuals to have an energy efficient pattern. In the human approach, "understanding how and why people use energy plays a role in achieving energy conservation" [3].

By using basic statistical analysis tools, the basic information about the energy usage patterns can be extracted. This information is later used as input for further, more detailed analysis, using more sophisticated tools and techniques.

\section{A. Collecting Energy Consumption Data}

Today's approach to data collection in the households is done by using smart digital devices that automatically measure energy usage in regular intervals. The collected data (the datasets) will contribute to find and quantify energysaving opportunities.

Lately, various datasets for energy consumption in households have been published. Most of these datasets that we have observed, such as BLUED, REDD, Smart and GREEND [4], record data in short-term measurement periods, such as less than a year. Additionally, these datasets do not collect energy consumption of all of the appliances and they do not capture various power measurement units (voltage, current, frequency, real power etc.).

We need to mention that some of the datasets monitor different households over different time intervals, and this aspect gave additional complexity for comparing them [4].

Based on the previously mentioned criteria we have analysed the characteristics of the following datasets: ECO data set [5], UK-DALE [6] and AMPds dataset [7].

The ECO dataset consists of fine-grained electricity consumption data for 5 households in Switzerland during a period of about 8 months [5]. It collects aggregated electricity consumption data at $1 \mathrm{~Hz}$ and the measurement contains information on voltage, current and phase shift between voltage and current [5]. The performance analysis of the algorithms used in ECO data set, have been proved as inadequate for real word applications [5]. Another dataset that covers a long span of electricity data measurement (a period of 499 days) is the UK-DALE [6]. This dataset contains both the reactive and real power recordings from 4 household appliance-detailed electricity usage levels [6]. To this moment, we have found only one dataset that contains detailed measurements for a period of two years; the AMPds dataset. This dataset has 60 seconds recording intervals about energy consumption of a single-house located in Greater Vancouver [7]. Apart from electrical power consumption, the AMPds dataset also provides detailed information for gas and water consumption in numerous measurements units.

\section{B. Basic Analysis of the Dataset}

For the purpose of this research we have decided to run analysis on the AMPds dataset by using standard statistical techniques to evaluate the electricity used during a period of two years.

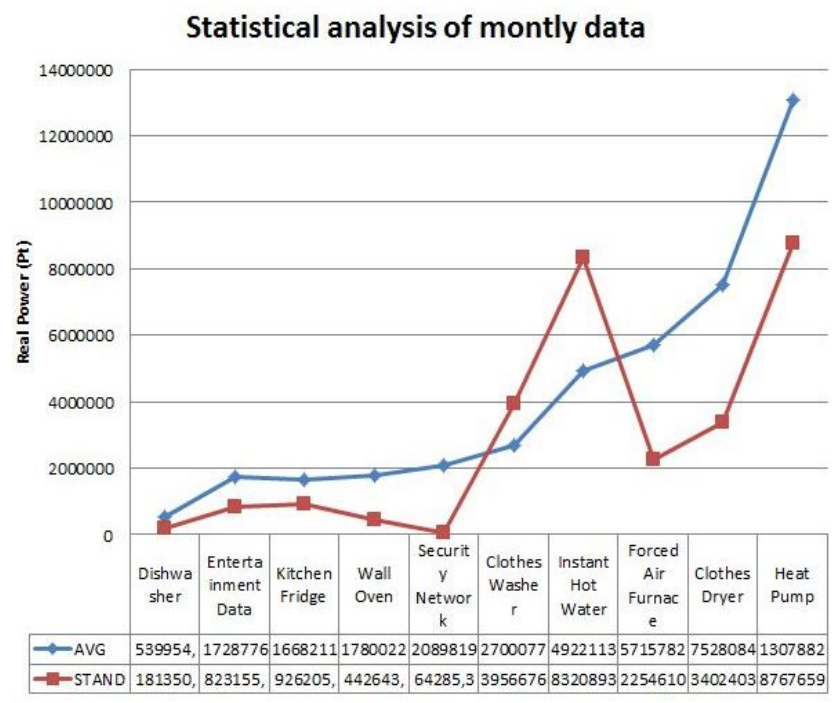

Fig. 1. Statistical analysis of monthly aggregated data.

Firstly, we identified ten most commonly used household appliances. For these ten devices, we have calculated the energy spent in watts, as products of the measured voltage and current $(\mathrm{W}=\mathrm{V} \times \mathrm{I})$. As it was previously mentioned, the metering interval is per minute.

We aggregated the raw dataset of AMPds to hourly, daily, monthly and total consumption data.

After that, basic statistical analysis was performed, such as mean value and standard deviation for the aggregated monthly data. This analysis has confirmed that the heat 
pump and clothes dryer are spending most of the energy on a monthly basis (see Fig. 1). These parameters bring to our attention that the household had increased usage of these appliances in the mentioned period. This triggered the idea about the problem of proper and optimal usage of some household appliances.

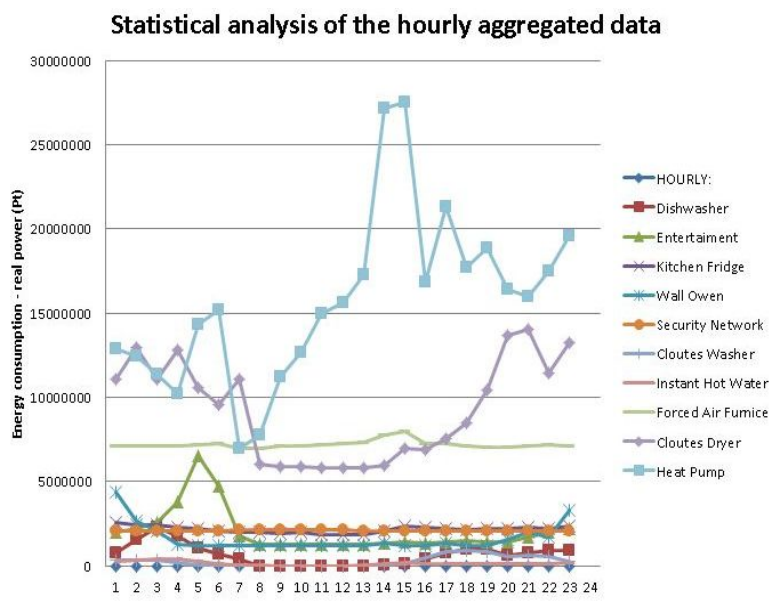

Fig. 2. Statistical analysis of hourly aggregated data.

Finally, the statistical analysis of the hourly aggregated data had led us to the discovery of the daily trend of energy consumption in the household. In Fig. 2, we notice the highest peak of energy spending during the period between 14 hours and 15 hours for the heat pump.

\section{Case study: Linear Regression Analysis of a Heat Pump}

After the first data analysis from the dataset, we have concluded that the main energy consumer is the heat pump. This consumption is correlated with the weather conditions in that area which imposes a need for further analysis. Regression analysis is typically a good option for analysis of this data [8], [9].

To correlate the energy usage with the outside air temperature, we are using the concept of degree days, which are a simplified form of historical weather data [2].

The degree data for the purposes of this paper are downloaded from [10]. The degree days can be: Heating (HDD) or Cooling (CDD). For the purposes of our research we have used the heating degree days, because they are related with the heating of buildings. "HDD figures come with a "base temperature", and provide a measure of how much (in degrees) and how long (in days), the outside temperature was below the base temperature" [2].

The data in the AMPds dataset that we have analysed are collected from the area of Greater Vancouver, British Columbia. Therefore, we have downloaded degree days from the station in that area. The regression analyses are done for two different periods (01.04.2012 - 01.04.2013) and (01.04.2013 - 01.04.2014), in order to find out any correlation changes in energy saving. For our analysis, we choose the base temperature to be $19.5^{\circ} \mathrm{C}$. According to the conclusion from the provider of the AMPds dataset [7], we are using Current (I) instead of Real Energy (Pt) for the regression analysis.

The regression lines (Fig. 3 and Fig. 4), give a relationship between degree days and the Current (I) for each period respectively.

\section{Regression analysis for the period of 04.2012-03.2013}

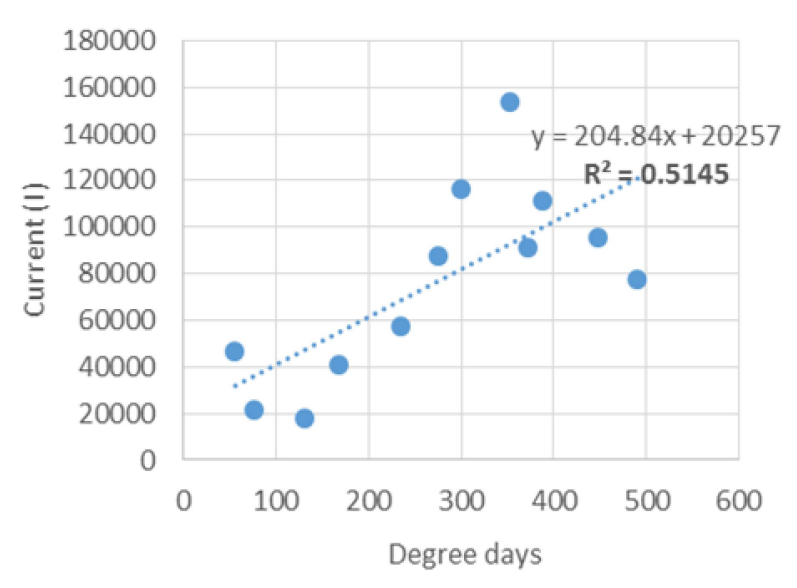

Fig. 3. Regression analysis for the period of $04.2012-03.2013$.

The $\mathrm{R}^{2}$ value indicates how good the correlation is between energy use and degree days. The best correlation is achieved when $\mathrm{R}^{2}$ approaches 1 . If the value of $\mathrm{R}^{2}$ is much below 0.7 , that indicates that the heating control is poor (assuming that the analysis methodology is good).

\section{Regression analysis for the period of $04.2013-03.2014$}

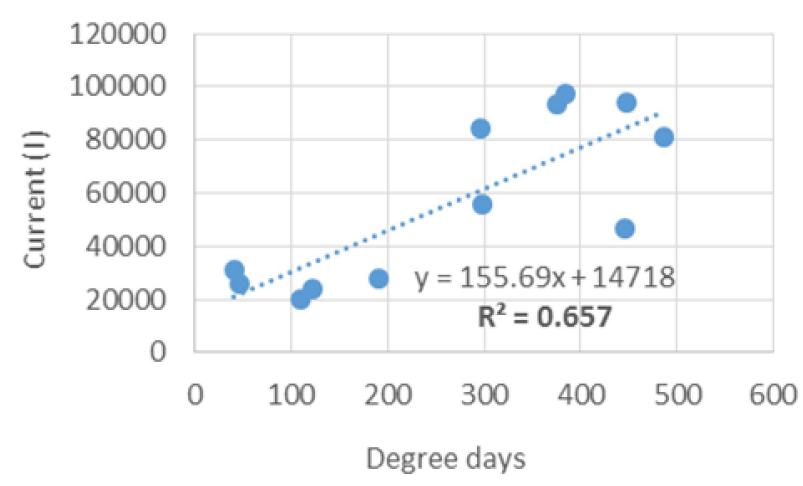

Fig. 4. Regression analysis for the period of $04.2013-03.2014$.

In our case, for both periods the correlation is poor, but there is some improvement in the correlation for the second period (Fig. 4). The conclusion is that some more actions should be taken for further improvement of the correlation, which will result in more energy saving. Those actions can be either buying new equipment, or improving the building's insulation, or causing additional behavioural changes.

\section{OVERVIEW OF ADVANCED TECHNIQUES FOR DATA ANALYSIS}

The statistical approach to energy consumption data implies the need of more advanced techniques for data analysis and prediction of future trends of energy consumption that should lead to optimization.

The existing research done in the area of smart energy consumption systems had used various algorithms to analyse gathered collections of datasets. There have been proposed smart metering models for monitoring the energy consumption in households and buildings, such as the NILM (Non-intrusive load monitoring) system [5]. Author S. Makoni in [7], had used "single-measurement disaggregation algorithm, by using discrete probabilities and single measurement, in order to disaggregate four types of 
appliances (simple on/off, finite-state, constantly on and continuously variable)". Also, it is very common to notice the widely known data mining algorithms, such as Clustering and Classification [11], which can be used to predict the energy consumption. The Inter-Dependency clustering model has been proposed to predict the energy consumption of different home appliances [12].

One of the popular algorithms used in Data mining is Evolutionary algorithm [13], [14] that is an umbrella term for bio-inspired algorithms [15], [16] and swarm algorithms like Artificial Bee's Colony Algorithm (ABC algorithm) [17], [18], Ant colony optimization [19] or Bacterial Colony Optimization [20]. More recently, we have found "an intelligent data-analysis system that had proven the use of the (Artificial Bee Colony) ABC algorithm as a new tool for Data Mining particularly in classification tasks" [21]. Considering that the classification tasks are necessary to extract valuable information from the large datasets, we had considered the $\mathrm{ABC}$ algorithm as a starting base for building our proposed Smart-ECO model.

\section{The SMART ECO MOdeL}

After some research on optimization algorithms, we have decided to take on an approach of the Artificial Bee's Colony Algorithm (ABC algorithm) [17], [18], [22], [23] with modifications to suite our particular purposes, namely, at the end of an optimization cycle (per 60 seconds) we should have all currently "on" devices (non-idle devices) categorized into 3 classes.

The classical bee algorithm is one which is based on the inherent ability of bees to optimize their swarming patterns while scouting for nectar. Ideally, after each iteration of an optimization cycle, the result is more optimized than the previous cycle, so there is a convergence towards the most optimum solution [22].

\section{The ABC algorithm}

$n$ - Number of scout bees

$m$ - Number of sites

$e$ - number of best sites out of m selected visits

nep - number of bees recruited for best e sites

$n s p$ - number of bees recruited for the other (m-e) selected sites

$n g h$ - initial size of patches

1. Start

2. Initialize Population

3. Evaluate fitness of population

4. While (stopping criteria is not met)

5. Select Sites for neighborhood search

6. Recruit bees for selected sites (more bees for best e sites) and evaluate fitness

7. Select the fittest bee from each patch

8. Assign remaining bees to search randomly and evaluate their fitness

9. Check ending criteria

End

This algorithm begins with initializing the bees (n) into randomly positions in the search space $(\mathrm{m})$. The viability (fitness) of the sites is than evaluated by the scout bees. Once the fitness of a site has been evaluated, the best sites are picked. The neighbouring sites (to the picked ones) are then evaluated (steps 6 and 7). Usually, the fitness values of the new sites are used to determine how many bees are recruited to scout the neighbouring sites. Therefore, sites that neighbour highly fit sites are usually assigned more bees to be evaluated by. Any remaining bees are assigned to a random search pattern (step 8). All of these steps are then repeated until a stopping criterion is met.

In our case, we want to obtain a periodic list of devices with their current wattage being used. This would then be optimized and categorized as necessary.

The Smart ECO (Electricity Consumption Optimization) algorithm, as we have named this modification of the ABC algorithm, is based on a behavioural approach which is crucial in defining habits. Habits are important in order to derive rules about the usage of home appliances. These rules are unique for every household which is a system for itself and has its own smart model. From this approach, we derive the concept of "habitual average" or average per habit as a central concept in the Smart ECO algorithm.

We define Habitual Average as a function of a few parameters: time, standard average and predefined usage

$$
H A=f(\text { timestamp, avg, dUsage }),
$$

where $H A$ - Habitual Average; timestamp - time of the day + full date; avg - standard/normal average for that timestamp (sum of elements over their count); dUsage - defined usage, the usage habits that the user entered when they configured the device (usually in hours).

We consider that this approach in defining an average has a better standpoint than the standard average definition as a sum of elements divided by their count. In order for the algorithm to be smart, it should have the capability of learning from the usage habits of the members of the household. However, learning only from the usage habits might be ambiguous because there should be some other figures considered which make up a big part of the optimization requirements.

The timestamp is important because it points to any dependencies there might be with regards to the defined usage and the time of use, in the sense of checking if the time of usage of a particular appliance deviates from the usual time of usage for that appliance, how usual is the behaviour of using a particular appliance at a particular timestamp, and also for deriving new rules and improving the learning process about habits of usage. This timestamp contains information about the full date and the time of the day at which the measurement was taken.

The standard average is useful to give basic information about any potential usage fluctuations from what is considered normal usage on average for that particular time of the day and year. In the case of big usage deviations from the normal average, further checks are performed by the algorithm in order to discover if this is a case of a new usage pattern or a case of a single special usage at the time.

Defined usage consists of information that the user defined themselves at the time of configuration of the smart device. This is usually expressed in hourly intervals which give information about what should be considered normal 
usage and in what period of the day and year. This information is collected in the form of poll questions that the smart device asks the user to answer, for example:

1. During what period of the year do you use your heating the most? (selection of months to make a month interval) While in heating usage months, during what period of the day are you using your heating the most? (selection of hours/time during the day to make a daily interval).

Questions of this type are prompted to the user for each appliance that is connected to the smart device and this information are used as a starting point for the learning phase of the algorithm and are treated as the primary definition of usage patterns. Considering all the hereby discussed parameters, the proposed Smart ECO algorithm would have the following pseudo format:

\section{The Smart ECO algorithm START \\ CLASSES: L, M, H \\ B: Send timestamp + Watts every $60 \mathrm{sec}$ (synchronous) Q:}

1. Check IF the sum of Watts deviates from an average (average is predefined with some tolerance) for that time of the day/month/year

Consider number of devices in use at the time

$\Rightarrow$ SEND NOTIFICATION immediately for big deviations

2. IF current usage is not below the average THEN Check what device is using most Compare device usage to usage habits

Based on this comparison, classify as L or $\mathrm{M}$ or $\mathrm{H}$

\section{REPEAT}

FOR ALL devices that are more than average based on habits, AND are classified as L for more than $30 \mathrm{~min}$

\section{$\Rightarrow$ SEND NOTIFICATION}

IF notification is ACCEPTED

optimize usage (turn device off for some time)

ELSE

END

ignore and continue

The main goal that the algorithm wants to achieve is to classify current appliance usage into one of three classes: $\mathrm{L}$ (low), $\mathrm{M}$ (middle) or $\mathrm{H}$ (high), where $\mathrm{L}, \mathrm{M}$ and $\mathrm{H}$ refer to low, middle and high priority of usage for the appliance at that time. After the usage of a particular appliance has been tracked over a certain period of time (e.g. 30 minutes), some decisions about notification sending can be made. The algorithm targets Low Priority classified appliances for sending optimization notifications/suggestions to the household. High Priority classified appliances are not targeted with notifications and the Middle class is used as a potential target (starting data) in the next iteration of the algorithm. Once L-notifications are sent to the household through the smart device, if the user accepts the optimization suggestion, the smart device would automatically act accordingly with the appliance in question, e.g. Smart device: I figured that your heater can be turned off for the next 10 minutes to optimize your electricity usage. Do you want me to do this? $(Y / N)$. IF $Y$-> Smart device turns off the heater for the next 10 minutes.

It is important to highlight here that this algorithm develops a capability to track new usage patterns outside of the set of initially user-defined usage patterns that the algorithm takes to start learning with. This is important in order to refine the sense about usage for each unique case of every household. For this purpose, any unusual usage is recorded temporarily and further investigated to find out if it was just a single usage deviation or maybe a lead to a new usage pattern. This behavioural tracking of potential new habits helps the algorithm update itself with new rules and provide higher-level of optimization with longer use of the smart device.

The Smart ECO model is complemented by its own Smart ECO hardware consisted of two types of sensors: a B-sensor and a Q-sensor (Fig. 5) [24], [25].

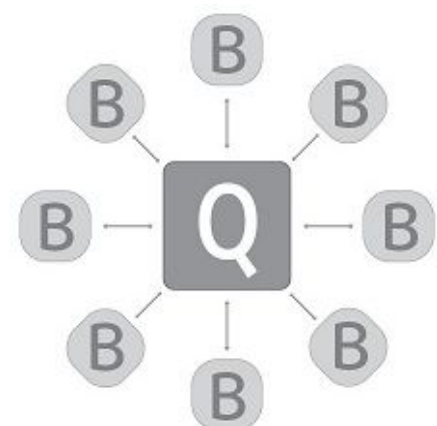

Fig. 5. Smart ECO Device Ecosystem - B-sensor and Q-sensor.

These devices use the analogy of the ABC Algorithm (Bees and Queen). The B-sensors are used as in-between plugs between the power socket and the appliance in question, and they send energy usage information about that particular appliance in regular 60-second intervals to the Qsensor. The Q-sensor is the central, wireless smart hub placed in the household, which communicates with the Bdevices in order to gather data and perform the Smart ECO algorithm on it. It can also send basic instructions to the Bsensor like switch on/off.

\section{CONCLUSIONS}

In this paper, we present a complete energy management process consisted of collection of energy consumption data and performing basic statistical analysis on the dataset. The analysis of the energy consumption data is done using the AMPds dataset. Based on the obtained results from this data manipulation, we bring some conclusions about the improvement of the energy consumption in the household Since the heat pump has proven to be the main energy consumer in the household, further analysis was conducted using the linear regression model. The purpose of this analysis is to discover if the currently used heating system by the household is in good function or needs improvements. The analysis has shown that the heating system used in that particular household is not a very good one, but there is a noticeable improvement in the more recent period.

The previously discussed analysis relied on the fact that the heating system is correlated with factors external to the household, namely, outside air temperature. Furthermore, in 
order to get a more refined sense of the factors that influence energy consumption, we considered looking at the internal household factors, the main ones being the behavioural patterns of the members of the household.

Based on this, we propose a new Smart ECO model for energy consumption optimization. The Smart ECO algorithm introduces a new concept called "Habitual Average", which differs from the standard average terms by being a function of the time at which an appliance is used, the regular usage habits in the household, and the standard average usage for that appliance at that time. The Smart ECO model makes use of its own smart devices for metering usage (B-sensor) and one central smart device that coordinates all, the Q-sensor. The main distinction of this smart model among other energy consumption optimization models is the per-appliance approach in order to provide a fine-grained optimization. This model is only a starting point to what should become a very refined Smart ECO model, ready to be deployed in smart devices and released for consumer use in real households. Further and more detailed approach to the Smart ECO model is to follow in a later discussion.

\section{REFERENCES}

[1] Smart cities: innovation in energy will drive sustainable cities. 2013. [Online] Available: http://www.theguardian.com/sustainablebusiness/smart-cities-innovation-energy-sustainable, accessed 02.12.2014

[2] Energy Lens - Energy Management Software. [Online] Available: http://www.energylens.com/, accessed 20.11.2014

[3] N. Khansari, A. Mostashari, M. Mansouri, "Conceptual modeling of the impact of smart cities on household energy consumption", in Conf. on Systems Engineering Research (CSER 2014), 2014. [Online] Available: http://dx.doi.org/10.1016/j.procs.2014.03.011

[4] A. Monacchi, D. Egarter, W. Elmenreich, S. D’Alessandro, A. M. Tonello, "GREEND: An energy consumption dataset of households in Italy and Austria", in Proc. 5th IEEE Int. Conf. Smart Grid Communications (SmartGridComm 2014), 2014, Venice, Italy. [Online]. Available: http://dx.doi.org/10.1109/smartgridcomm.2014. 7007698

[5] C. Beckel, W. Kleiminger, R. Cicchetti, T. Staake, S. Santini, "The ECO data set and the performance of non-intrusive load monitoring algorithms", in Proc. 1st ACM Conf. on Embedded Systems for Energy-Efficient Buildings (BuildSys 2014), New York, NY, USA 2014, pp. 80-89. [Online] Available: http://dx.doi.org/10.1145/ 2674061.2674064

[6] J. Kelly, W. Knottenbelt, "UK-DALE: A dataset recording UK Domestic Appliance-Level Electricity demand and whole-house demand", in ArXiv e-prints, 2014.

[7] S. Makonin, F. Popowich, L. Bartram, B. Gill, I. V. Bajic, "AMPds: A public dataset for load disaggregation and eco-feedback research", in IEEE Electrical Power \& Energy Conf. (EPEC), 2013, 2013, pp. 1-6. [Online] Available: http://dx.doi.org/10.1109/EPEC. 2013.6802949

[8] M. R. Braun, H. Altan, S. B. M. Beck, "Using regression analysis to predict the future energy consumption of a supermarket in the UK", Applied Energy, vol. 130, pp. 305-313, 2014. [Online]. Available: http://dx.doi.org/10.1016/j.apenergy.2014.05.062

[9] W. Tian, J. Song, Z. Li, "Spatial regression analysis of domestic energy in urban areas", Energy, vol. 76, pp. 629-640, 2014. [Online] Available: http://dx.doi.org/10.1016/j.energy.2014.08.057

[10] Degree Days.net [Online] Available: degreedays.net

[11] I. Khan, A. Capozzoli, S. P. Corgnati, T. Cerquitelli, "Fault detection analysis of building energy consumption using data mining techniques", Energy Procedia, vol. 42, pp. 557-566, 2013. [Online] Available: http://dx.doi.org/10.1016/j.egypro.2013.11.057

[12] N. C. Truong, J. McInerney, L. Tran-Thanh, E. Costanza, S. D Ramchurn, "Forecasting multi-appliance usage for smart home energy management", in 23rd Int. Joint Conf. Artificial Intelligence (IJCAI 2013), Beijing, CN, 2013.

[13] T. Back, Evolutionary algorithms in theory and practice: evolution strategies, Evolutionary Programming, Genetic Algorithms. Oxford Univ. Press, 1996.

[14] D. Simon, Evolutionary Optimization Algorithms: Biologically Inspired and Population-Based Approaches to Computer Intelligence. Wiley, 2013.

[15] S. Gao, Bio-Inspired Computational Algorithms and Their Applications. InTech, 2012. [Online]. Available: http://dx.doi.org/10.5772/2358

[16] S. Olariu, A.Y. Zomaya, Handbook of Bioinspired Algorithms and Applications. Chapman and Hall/CRC, 2005.

[17] D. T. Pham, A. Ghanbarzadeh, E. Koc, S. Otri, S. Rahim, M. Zaidi, "The Bees Algorithm - A Novel Tool for Complex Optimization Problems, in Intelligent Production Machines and Systems", in 2nd $I * P R O M S$ Virtual Int. Conf., 2006. [Online] Available: http://dx.doi.org/10.1016/B978-008045157-2/50081-X

[18] D. T. Pham, A. Ghanbarzadeh, E. Koc, S. Otri, S. Rahim, M. Zaidi, The bees algorithm. technical note, manufacturing engineering centre. Cardiff University, UK, 2005.

[19] M. Dorigo, T. Stutzle, Ant Colony Optimization, A Bradford Book, 2004.

[20] B. Niu, H. Wang, "Bacterial colony optimization: principles and foundations", in 8th In. Conf., (ICIC 2012), Huangshan, China, 2012. [Online] Available: http://dx.doi.org/10.1007/978-3-642-31837-5_73

[21] A. M. Shukran, Y. Y. Chung, W.-C. Yeh, N. Wahid, A. M. A. Zaidi, "Artificial bee colony based data mining algorithms for classification tasks", Modern Applied Science, vol. 5, no. 4, pp. 217-231, 2011. [Online]. Available: http://dx.doi.org/10.5539/mas.v5n4p217

[22] D. T. Pham, A. Ghanbarzadeh, E. Koc, S. Otri, S. Rahim, M. Zaidi, "The bees algorithm - a novel tool for complex optimization problems", in Proc. 2nd Int. Virtual Conf. Intelligent Production Machines and Systems, 2006. [Online]. Available: http://dx.doi.org/10.1016/B978-008045157-2/50081-X

[23] D. Karaboga, B. Basturk, "On the performance of artificial bee colony (ABC) algorithm”, Applied Soft Computing, vol. 8, no. 1, pp. 687-697, 2008. [Online]. Available: http://dx.doi.org/10.1016/ j.asoc.2007.05.007

[24] Wang Zhong-qin, Ye Ning, Reza Malekian, Zhao Ting-ting, Wang Ru-chuan, "Measuring the Similarity of PML Documents with RFIDbased Sensors", International Journal of Ad Hoc and Ubiquitous Computing, vol. 17, no. 3, 2014. [Online]. Available: http://dx.doi.org/10.1504/ijahuc.2014.065764

[25] Ning Ye, Yan Zhu, Ru-Chuan Wang, Reza Malekian, Lin Qiaomin, "An Efficient Authentication and Access Control Scheme for Perception Layer of Internet of Things", Applied Mathematics and Information Sciences, vol. 8, no. 4, pp. 1617-1624, 2014. [Online]. Available: http://dx.doi.org/10.12785/amis/080416 